\title{
Miscibility Behavior of Ethylene/Vinyl Acetate and C5 Petroleum Resin by FTIR Imaging
}

\author{
Xin Zhou, Pudun Zhang, ${ }^{\dagger}$ Zhifa LI, and Guoying Rao \\ School of Science, Beijing University of Chemical Technology, Beijing 100029, China
}

\begin{abstract}
FTIR microscopic imaging was used to investigate the miscibility behavior of ethylene/vinyl acetate copolymer (EVA) and $\mathrm{C} 5$ petroleum resin. Images with an area of $500 \times 500 \mu \mathrm{m}^{2}$ were collected in the reflection mode. The miscibility was characterized by probing the spatial distribution of the carbonyl group $(\mathrm{C}=\mathrm{O})$ of EVA in the whole images. It was found that a 1:1 hot-melt mixture of EVA and C5 resin showed a good miscibility behavior. For two different EVA copolymers, one with $18 \%$ vinyl acetate (VAc) content showed a better miscibility behavior than that with $28 \%$ VAc content. Our results demonstrated that this method allowed a direct, convenient and nondestructive visualization. This developed technique promises to become a powerful tool for studying the miscibility behavior of composite materials.
\end{abstract}

(Received March 7, 2007; Accepted April 9, 2007; Published July 10, 2007)

\section{Introduction}

Blending is an important approach to develop new polymer materials. The miscibility of the blends has a great influence on their performances, such as the phase structure, physical properties and some other bulk properties in practical use. Miscibility is essentially the ability to form a homogeneous system of two or more polymers. It is generally characterized by differential scanning calorimetry (DSC), ${ }^{1-6}$ scanning or transmission electron microscopy (SEM and TEM), ${ }^{1,2}$ Fouriertransform infrared spectroscopy (FTIR) ${ }^{4-6}$ and viscometry. ${ }^{1,6}$

FTIR microscopic imaging is a powerful tool developed during the last decade. ${ }^{7}$ Attempts to improve the technique have been made elsewhere. ${ }^{8-11}$ The image is obtained based on the distributions of chemical groups in a micro-region. It has been used to investigate biological and medical systems, ${ }^{12-15}$ biomaterials, ${ }^{16,17}$ artworks ${ }^{18,19}$ and polymer materials..$^{20-23}$ In the field of polymer science, this technique was used to characterize polymer blends, ${ }^{20,21}$ monitor the phase-separation process in producing polymer dispersed liquid crystal $\mathrm{s}^{22}$ and study polymer dissolution by solvent mixtures. ${ }^{23}$ Ethylene/vinyl acetate (EVA) hot melt adhesive (HMA) is a kind of widely used composite material because of its excellent adhesion and its non-pollution to the environment. ${ }^{24}$ It is generally composed of EVA resin, tackifier and liquid paraffin. Takemoto et al. ${ }^{25,26}$ investigated the relationship of the miscibility and adhesive properties of EVA-based HMA. The applied tackifiers included rosin-based, terpene-based and terpene phenolic resins. In the present work, we investigated the miscibility behavior of EVA and the tackifier C5 petroleum resin by applying FTIR imaging. The proposed method was direct and nondestructive visualization.

\section{Experimental}

EVA resins with two vinyl acetate (VAc) contents (EVA1 with

$\dagger$ To whom correspondence should be addressed.

E-mail: zhangpd@mail.buct.edu.cn
$18 \%$ and EVA2 with 28\%) were provided from the China market. C5 petroleum resin was kindly donated by Lanzhou Petrochemical Research Institute, PetroChina Co. Ltd. (Lanzhou, China). At the beginning, 30 parts by weight of liquid paraffin (chemical grade) were mixed with various parts $(50,75,100,150$ and 200) of C5 resin. The mixtures were then heated to $175^{\circ} \mathrm{C}$ under agitation until they became yellowish and transparent. Then, 100 parts of EVA resin were added into a hot solution and the temperature was kept at $175^{\circ} \mathrm{C}$ until it was absolutely dissolved. By maintaining agitation for about 30 min, an apparently homogeneous and viscous solution was finally formed. It was cooled down to room temperature, and the mixture became a hard, ivory-white solid.

A freezing microtome (YD-1508B, Jinhua YIDI Medical Facilities Manufactory, Zhejiang, China) was used to prepare a sample slice. A piece of HMA solid (about $1.0 \times 1.0 \mathrm{~cm}^{2}$ ) was fixed on the cryo-stage using deionized water by freezing to $-25^{\circ} \mathrm{C}$. It was then sectioned to $25 \mu \mathrm{m}$ thickness with a stainless-steel knife, which was also frozen to less than $-5^{\circ} \mathrm{C}$.

The FTIR spectra were collected using a Nexus 8700 FTIR spectrometer with a room-temperature deuterated triglycine sulfate (DTGS) detector (Thermo Electron Corp., WI, USA). FTIR images were acquired using the same spectrometer equipped with a Continuum XL Microscope with a 32-element $(16 \times 2)$ mercury cadmium tellium $(\mathrm{MCT})$ array detector and a MCT-A single detector (Thermo Electron Corp.). The prepared sample film was carefully spread on a gold-coated glass, and then the glass was placed on a microscopic stage. An IR beam was focused on the film by using a $15 \times$ objective with NA 0.58 . The imaging procedure was carried out in the reflection mode with a $8-\mathrm{cm}^{-1}$ spectral resolution and 8 scans for each fixed-size step. The image area was $500 \times 500 \mu^{2}$ and the total acquisition time was less than $3 \mathrm{~min}$.

\section{Results and Discussion}

The IR spectra of the three components are shown in Fig. 1. The spectrum of C5 petroleum resin (Fig. 1b) was similar to that of liquid paraffin (Fig. 1a). The characteristic peaks for both 


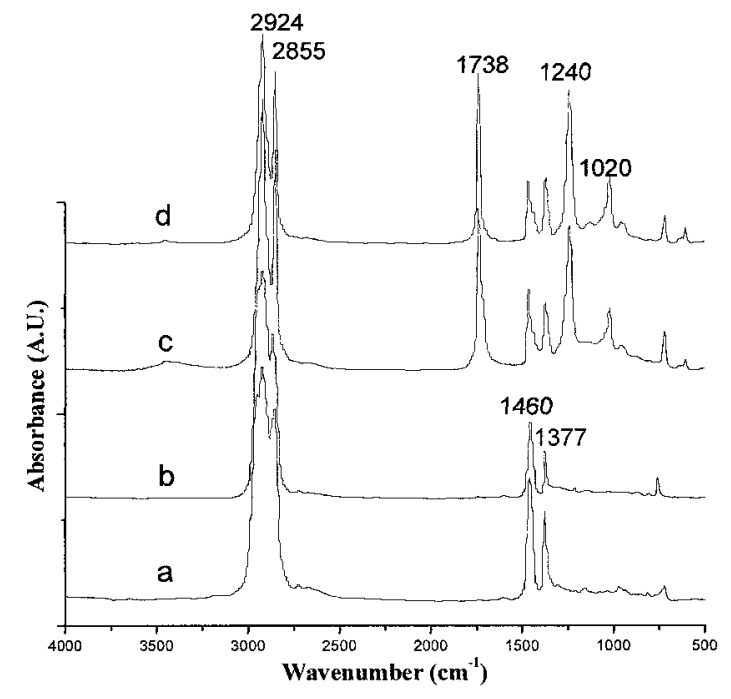

Fig. 1 FTIR spectra of (a) liquid paraffin, (b) C5 petroleum, (c) EVA1 with $18 \%$ VAc content and (d) EVA2 with $28 \%$ VAc content.

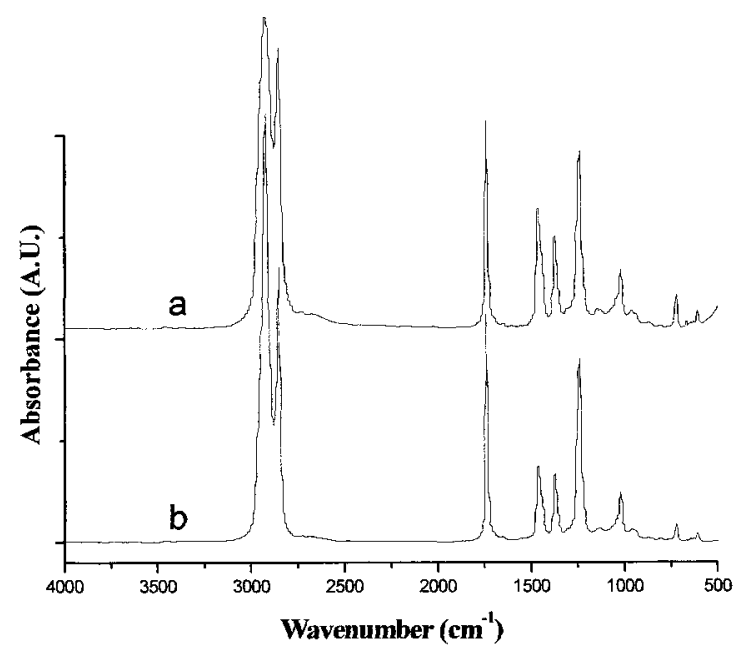

Fig. 2 FTIR spectra of (a) EVA1-based and (b) EVA2-based HMA.

were that at 2924 and $2855 \mathrm{~cm}^{-1}\left(v_{\mathrm{s}}\left(\mathrm{CH}_{2}\right)\right.$ and $\left.v_{\text {as }}\left(\mathrm{CH}_{2}\right)\right), 1460$ and $1377 \mathrm{~cm}^{-1}\left(\delta_{\mathrm{s}}\left(\mathrm{CH}_{2}\right)\right.$ and $\left.\delta_{\text {as }}\left(\mathrm{CH}_{2}\right)\right)$. For EVA resin (Figs. 1c and 1d), besides these 4 peaks, those at 1738, 1240 and 1020 $\mathrm{cm}^{-1}$ were caused by a stretching vibration of the carbonyl group $(\mathrm{C}=\mathrm{O})$ and the ester group $(\mathrm{C}-\mathrm{O})$, respectively. Although some slight changes $\left(0-4 \mathrm{~cm}^{-1}\right)$ in the peak position were observed, they could not in fact be discriminated for different materials, because they were beyond the experimental spectral resolution. We noted that for EVA2 the intensity of the peak at $1377 \mathrm{~cm}^{-1}$ was higher than that at $1460 \mathrm{~cm}^{-1}$. This is a consequence of the higher content of VAc units in EVA2, which contribute more methyl groups $\left(\mathrm{CH}_{3}\right)$ to the copolymer. The spectra of the two HMAs were similar (Fig. 2), and it was difficult to distinguish them using the traditional FTIR.

To characterize the miscibility, FTIR microscopic imaging was used. Liquid paraffin was expected to disperse in all of the domains due to its liquid state and the low molecular weight, and was thus not considered. Because the characteristic peaks of C5 (2924, 2855, 1460 and $\left.1377 \mathrm{~cm}^{-1}\right)$ are superimposed by those of EVA, it is difficult to explore the miscibility based on them. In this paper, the miscibility of EVA and C5 was revealed by studying the variation of the peak absorbance at

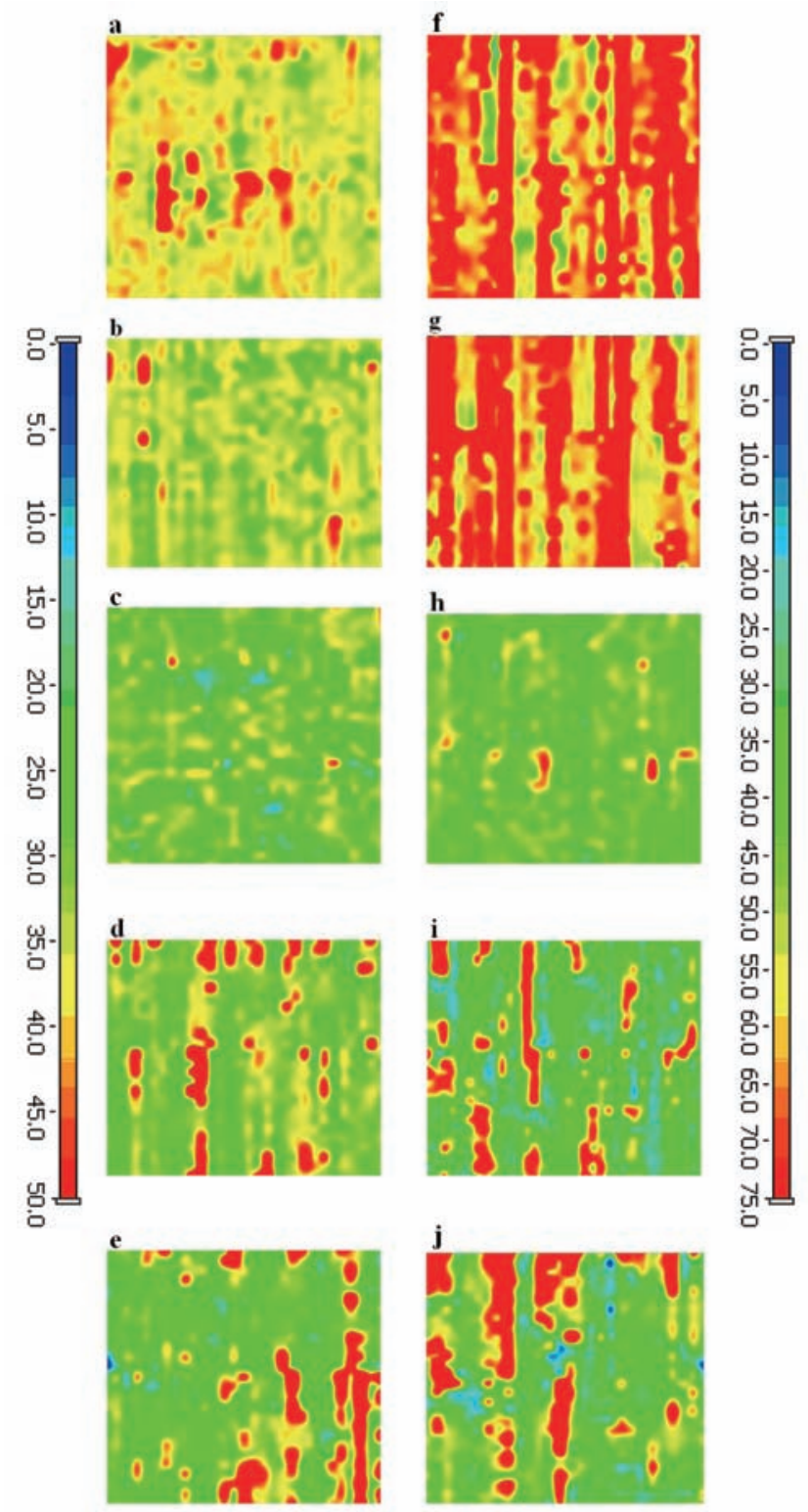

Fig. 3 FTIR images of HMAs of EVA to C5 resin at the proportion of (a and f) 100:50, (b and g) 100:75, (c and h) 100:100, (d and i) 100:150 and (e and j) 100:200 based on the carbonyl absorption band at $1738 \mathrm{~cm}^{-1}$. Color scale bars for EVA1-based (a to e) and EVA2based (f to $\mathrm{j}$ ) are given on the left and right side, respectively. The size of each image was $500 \times 500 \mu \mathrm{m}^{2}$.

$1738 \mathrm{~cm}^{-1}$ in different regions, which represented the spatial distribution of EVA. The more homogeneous was the distribution, the better was the miscibility. Since the composite material is a two-component system (not considering liquid paraffin), the distribution of C5 was expected to be complementary to EVA, although it could not be visualized in the FTIR image.

Figure 3 shows a series of FTIR images of EVA-based HMAs at different proportions of EVA to C5. Figures 3 a to 3e resulted from EVA1 and C5 resin, while Figs. $3 f$ to $3 j$ are from EVA2 and C5 resin. All of the images were based on the peak absorbance at $1738 \mathrm{~cm}^{-1}$, and were corrected by subtracting the baseline between 1800 and $1677 \mathrm{~cm}^{-1}$. The color in the images, which represented the intensity of the peak at $1738 \mathrm{~cm}^{-1}$, could be varied at any scales to highlight the chemical nonuniformity. It showed that EVA existed in most of the areas of the sample 
(a)
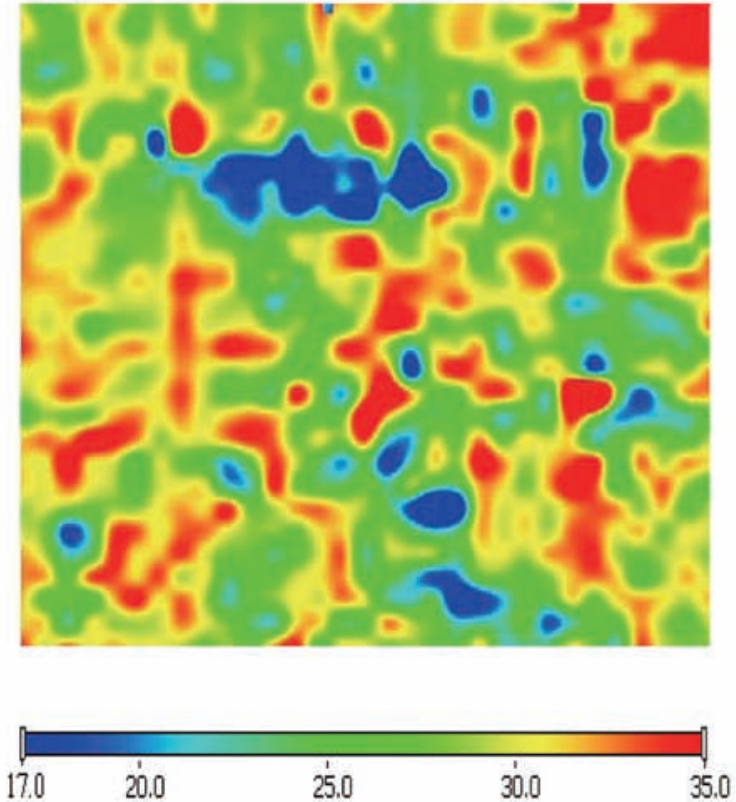

(b)
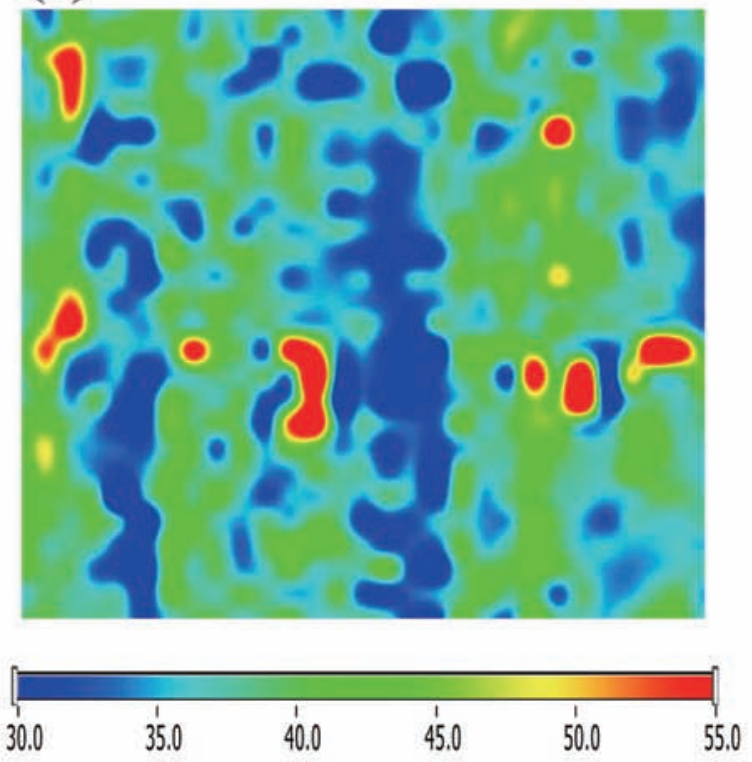

Fig. 4 FTIR image (a) and (b) for enhancing the contrast of Figs 3(c) and 3(h) by changing the color scale bar, respectively.

films. This suggested a good performance of these composite materials. Nevertheless, it was noticed that some areas had a dominant presence of EVA (red area in the image), which meant that a partial immiscible behavior was still present. It is indicated from Figs. $3 \mathrm{a}$ to $3 \mathrm{j}$ that the red area in EVA2-based HMA was always larger than that in EVA1-based HMA at the corresponding proportion. This was true because of a higher VAc content in EVA2.

It is also revealed that from Figs. 3a to 3e HMA of a 1:1 mixture of EVA1 to C5 (Fig. 3c) exhibited the best miscibility behavior. The other HMAs with various ratios of EVA1 to C5 would produce some domains of high or low content of the carbonyl group. Similarly, the result that HMA of 1:1 mixture of EVA2 to C5 resin (Fig. 3h) showed the best miscibility was also achieved from Figs. $3 \mathrm{f}$ to $3 \mathrm{j}$. Changes in the color scale did not affect the estimation. These results suggested that a good miscibility behavior can be obtained by mixing EVA and $\mathrm{C} 5$ resin at a proportion of one to one.

Consequently, we tried to compare Figs. $3 \mathrm{c}$ with $3 \mathrm{~h}$. We noticed that for Fig. $3 \mathrm{c}$ the dominant region (green area) was in the color range from 17.0 to 35.0 , while for Fig. $3 \mathrm{~h}$ it varied from 30.0 to 55.0. To enhance the image contrast, the color scale of the two figures was limited in the above ranges, respectively. In Fig. 4a the blue area (less than 23) and the red area (larger than 30) were randomly dispersed in the green area $(23-28)$. Further, the area ratio of red to green was approximately $1: 1$, which implied a homogeneous distribution of EVA1 in the HMA. On the other hand, in Fig. 4b there existed two distinct blue strips with a width of 50-100 $\mu \mathrm{m}$, which implied a relatively low content of EVA2 in the two strips of the HMA. The results showed that HMA of the 1:1 mixture of EVA1 to $\mathrm{C} 5$ resin showed a better miscibility behavior than that of EVA2 to C5.

\section{Conclusions}

In the present communication we developed a visualizing method to study the miscibility behavior of EVA and C5 petroleum resin by using FTIR imaging. The images were constructed in terms of the spatial distribution of the carbonyl peak in a given region. The color in the images reflected the EVA content in the corresponding position. The miscibility behavior was estimated based on the homogeneity of the color distribution. Our results showed that a good miscibility behavior was obtained when EVA was mixed with $\mathrm{C} 5$ resin at a proportion of one to one. We also proved that a better miscibility behavior was acquired for EVA with $18 \%$ VAc content than that with $28 \%$ VAc content. Although it was applied for studying the miscibility behavior of EVA-based HMAs in this paper, we definitely point out that this attractive technique can be used for any kind of composite materials, provided that one or more components have characteristic absorption bands in the IR region.

\section{Acknowledgements}

We are grateful to Lanzhou Petrochemical Research Institute, PetroChina Co. Ltd. for the donation of the $\mathrm{C} 5$ petroleum resin.

\section{References}

1. O. Monticelli, S. Russo, R. Campagna, and B. Voit, Polymer, 2005, 46, 3597.

2. P. S. O. Patricio, J. A. de Sales, G. G. Silva, D. Windmoller, and J. C. Machado, J. Membr. Sci., 2006, 271, 177.

3. C. M. Zaccaron, R. V. B. Oliveira, M. Guiotoku, A. T. N. Pires, and V. Soldi, Polym. Degrad. Stab., 2005, 90, 21.

4. E. Meaurio, E. Zuza, and J.-R. Sarasua, Macromolecules, 2005, 38, 9221.

5. K. Lewandowska, Eur. Polym. J., 2005, 41, 55.

6. K. Aouachria and N. Belhaneche-Bensemra, Polym. Test., 2006, 25, 1101.

7. E. N. Lewis, P. J. Treado, R. C. Reeder, G. M. Story, A. E. Dowrey, C. Marcott, and I. W. Levin, Anal. Chem., 1995 , 67, 3377. 
8. C. M. Snively and J. L. Koenig, Appl. Spectrosc., 1999, 53, 170.

9. R. Bhargava, T. Ribar, and J. L. Koenig, Appl. Spectrosc., 1999, 53, 1313.

10. K. L. A. Chan and S. G. Kazarian, Appl. Spectrosc., 2003, 57,381 .

11. E. Levenson, P. Lerch, and M. C. Martin, Infrared Phys. Tech., 2006, 49, 45.

12. R. Mendelsohn, E. P. Paschalis, P. J. Sherman, and A. L. Boskey, Appl. Spectrosc., 2000, 54, 1183.

13. K. Potter, L. H. Kidder, I. W. Levin, E. N. Lewis, and R. G. S. Spencer, Arth. Rheum., 2001, 44, 846.

14. A. Naumann, M. Navarro-Gonzalez, S. Peddireddi, U. Kues, and A. Polle, Fungal Genet. Biol., 2005, 42, 829.

15. E. Y. Jiang and J. Rieppo, J. Mol. Struct., 2006, 799, 196.

16. S. G. Kazarian, K. L. A. Chan, V. Maquet, and A. R. Boccaccini, Biomaterials, 2004, 25, 3931.

17. X. Bi, X. Yang, M. P. G. Bostrom, and N. P. Camacho,
BBA-Biomembranes, 2006, 1758, 934.

18. K. Keune and J. J. Boon, Anal. Chem., 2004, 76, 1374.

19. A. van Loon and J. Boon, Spectrochim. Acta, Part B, 2004, $59,1601$.

20. S. J. Oh and J. L. Koenig, Anal. Chem., 1998, 70, 1768.

21. A. Gupper, P. Wilhelm, M. Schmied, S. G. Kazarian, K. L. A. Chan, and J. Reussner, Appl. Spectrosc., 2002, 56, 1515.

22. R. Bhargava, S.-Q. Wang, and J. L. Koenig, Macromolecules, 1999, 32, 2748.

23. B. A. Miller-Chou and J. L. Koenig, Macromolecules, 2002, 35, 440.

24. Y.-J. Park, H.-S. Joo, H.-J. Kim, and Y.-K. Lee, Int. J. Adhes. Adhes., 2006, 26, 571.

25. M. Takemoto, M. Kajiyama, H. Mizumachi, A. Takemura, and H. Ono, J. Appl. Polym. Sci., 2002, 83, 719.

26. M. Takemoto, M. Kajiyama, H. Mizumachi, A. Takemura, and H. Ono, J. Appl. Polym. Sci., 2002, 83, 726. 